\title{
Single Biomolecule Imaging by Electrochemiluminescence
}

\author{
Yujie Liu,${ }^{\dagger}$ Hongding Zhang, ${ }^{\dagger}$ Binxiao Li,${ }^{\dagger}$ Jianwei Liu,${ }^{\dagger}$ Dechen Jiang, ${ }^{*}, \|$ Baohong Liu ${ }^{*},{ }^{\dagger}$, Neso Sojic ${ }^{*}, \dagger$
}

†Department of Chemistry, Shanghai Stomatological Hospital, State Key Laboratory of Molecular Engineering of Polymers, Fudan University, Shanghai 200433, P. R. China

tUniversity of Bordeaux, Bordeaux INP, ISM, UMR CNRS 5255, 33607 Pessac, France

"State Key Laboratory of Analytical Chemistry for Life and School of Chemistry and Chemical Engineering, Nanjing University, Nanjing, Jiangsu, 210093, P. R. China

\section{Table of Contents}

\section{Experimental Procedures}

Chemicals and Reagents

Synthesis of RuDSNs

Synthesis of RuDSN/AuNPs

Synthesis of RuDSN/AuNPs/Ab 2 Nanoemitters

Preparation of Aminated ITO

Preparation of ITO Electrode and Sample Cell

Fabrication Procedure of Biosensor

Single-Molecule Fluorescence Imaging

Culture and Fix Cells on the ITO Electrode

ECL Detection and Imaging

Data Analysis Process for Single Molecule Fluorescence Imaging

Estimation of the Average Number of Dyes per NP

\section{Supplementary Figures}

S1. Characterization of the Nanoemitter

S2. ECL Enhancement from RuDSN in presence of AuNPs

S3. Scheme Illustration for the Single Protein ECL Imaging

S4. Electrochemical and ECL Response of the Nanoemitter

S5. Voltage Optimization

S6. Frequency Optimization

S7. Exposure Time Optimization

S8. Size Analysis of PL and ECL spots

S9. The AFM image of ECL nanoemitters

$S 10$. The $S / N$ ratios of $E C L$ and $P L$ from single antigen

S11. Stability of ECL from single antigen

S12. Reproducibility and Stability of the Proposed Method

$\mathrm{S} 13$. The $\mathrm{S} / \mathrm{N}$ ratios of $E C L$ and $P L$ from the antigens at single cells

Table S1. Detection of CK19 in Human Serum

\section{References}




\section{Experimental Procedures}

\section{Chemicals and Reagents}

Triton X-100, Tween 20, tetraethyl orthosilicate (TEOS, $>99.9 \%$ ), cyclohexane, and $n$-hexanol were obtained from Aladdin Industrial Co., Ltd. (Shanghai, China). (3-Aminopropyl) triethoxysilane (APTES), chloroauric acid $\left(\mathrm{HAuCl}_{4} \cdot 4 \mathrm{H}_{2} \mathrm{O}\right)$, tris(2,2'-bipyridyl) ruthenium(II) chloride hexahydrate $\left(\mathrm{Ru}(\mathrm{bpy})_{3} \mathrm{Cl}_{2} \cdot 6 \mathrm{H}_{2} \mathrm{O}, 98 \%\right)$, tripropylamine (TPA), glutaraldehyde (GLD), bovine serum albumin (BSA, $\geq 98 \%$ ), poly-L-lysine (PLL), paraformaldehyde (PFA) were purchased from Sigma-Aldrich (St Louis, USA). Ascorbic acid, ammonia solution $\left(\mathrm{NH}_{3} \cdot \mathrm{H}_{2} \mathrm{O}, 30 \%\right)$, and hydrogen peroxide $\left(\mathrm{H}_{2} \mathrm{O}_{2}, 30 \%\right)$ were obtained from Sinopharm Chemical Reagent Co., Ltd. (Shanghai, China). Acetone and ethanol were obtained from Shanghai Dahe Chemical Co., Ltd. (Shanghai, China). Recombinant human keratin 19 (CK19, >90\%) and its mouse monoclonal antibodies (clone no. M210C and CY5\# as capture antibodies Ab ${ }_{1}$, M5B and $\mathrm{CY} 7 \#$ as detection antibodies $A b_{2}$ ) were purchased from Beijing Key-Bio Biotech Co., Ltd. (Beijing, China). Biotin AntiCytokeratin 19 antibody was obtained from Abcam Trading Co., Ltd (Shanghai, China). Cy5 conjugated with streptavidin was purchased from Invitrogen Co., Ltd (Carlsbad, CA, USA). Carcinoembryonic antigen (CEA), alpha fetoprotein (AFP), and prostate specific antigen (PSA) were obtained from Shanghai Solarbio Bioscience \& Technology Co., Ltd. (Shanghai, China). Dulbecco's modified eagle's medium (DMEM), fetal bovine serum (FBS), trypsin, and phosphate buffered saline (PBS) were purchased from Gibco (USA).

\section{Synthesis of RuDSNs}

RuDSNs were synthesized using the water-in-oil method according to the previous literature with some modifications..$^{1-3}$ First, 1.77 $\mathrm{mL}$ of Triton X-100 were mixed with $7.5 \mathrm{~mL}$ of cycloexane, $1.8 \mathrm{~mL}$ of $n$-hexanol, and $340 \mu \mathrm{L}$ of Ru(bpy) ${ }_{3}{ }^{2+}$ solution in water. After 30 min vigorous stirring, $100 \mu \mathrm{L}$ of TEOS was added into the above prepared water-in-oil emulsion. Then, a polymerization reaction was initiated by adding $60 \mu \mathrm{L}$ of $\mathrm{NH}_{3} \cdot \mathrm{H}_{2} \mathrm{O}$. The reaction was allowed to continue for $24 \mathrm{~h}$ at room temperature. Thereafter, acetone was added to break the microemulsion and recover the particles. The products were centrifuged and washed with ethanol and water several times to remove any surfactant molecules. At last, some completely dry orange RuDSNs were obtained.

\section{Synthesis of RuDSN/AuNPs}

To ensure that the surface of the RuDSNs contained amino groups, $3 \mathrm{mg}$ of RuDSNs were dispersed with $1.5 \mathrm{~mL}$ of ethanol and $50 \mu \mathrm{L}$ of APTES was added in the solution. After being stirred for $30 \mathrm{~min}$, the mixture was centrifuged and washed with ethanol to remove the excess APTES, and the amino-terminated RuDSNs were obtained and redispersed in $1.0 \mathrm{~mL}$ of water. Then, $500 \mu \mathrm{L}$ of $1 \% \mathrm{HAuCl}_{4} \cdot 4 \mathrm{H}_{2} \mathrm{O}$ was mixed with the above water solution and stirred vigorously for 10 min. Freshly prepared $530 \mu \mathrm{L}$ of $0.5 \mathrm{mM}$ ascorbic acid was added into the solution and followed by stirring for $5 \mathrm{~min}$. After centrifugation, the heterogeneous RuDSN/AuNPs were washed with water, centrifuged, and finally resuspended in $500 \mu \mathrm{L}$ of water.

\section{Synthesis of RuDSN/AuNPs/Ab 2 Nanoemitters}

$100 \mu \mathrm{L}$ of the RuDSN/AuNPs suspension was mixed with $100 \mu \mathrm{L}$ of the $\mathrm{Ab}$, solution $(100 \mu \mathrm{g} / \mathrm{mL}$ in $0.01 \mathrm{M}$ PBS, pH 7.4). Following incubation at $30^{\circ} \mathrm{C}$ for $3 \mathrm{~h}$, the solution containing $A b_{2}$ functionalized RuDSN/AuNPs (RuDSN/AuNPs/Ab $b_{2}$ ) was centrifuged and washed with PBS, and then redispersed in $0.01 \mathrm{M} \mathrm{PBS}(\mathrm{pH} 7.4)$ containing $0.05 \%$ Tween 20 and $0.01 \%$ BSA.

\section{Preparation of Aminated ITO}

ITO coated cover slips $(20 \times 20 \mathrm{~mm}, 0.17 \mathrm{~mm}$ thick) with $\sim 15-30 \Omega /$ square surface resistivity (SPI Supplies, USA) were cleaned with successively by sonication in acetone, ethanol, and water for $10 \mathrm{~min}$, and then dipped into a solution of 1:1:5 (v/v) $\mathrm{H}_{2} \mathrm{O}_{2} / \mathrm{NH}_{3} \cdot \mathrm{H}_{2} \mathrm{O} / \mathrm{H}_{2} \mathrm{O}$ for 30 min and followed by washing with water. Finally, the ITO slides were silanized with $1 \%$ APTES (ethanol) for $10 \mathrm{~s}$ and heated at $80^{\circ} \mathrm{C}$ for $30 \mathrm{~min}$. After incubation, the ITO slices were washed with water to remove the physically absorbed silanes from the ITO electrode surface. Thus amino groups were formed on the ITO electrode.

\section{Preparation of ITO Electrode and Sample Cell}

As seen in Figure S4, for the fabrication of the ITO electrode, a corner of the aminated ITO was connected to conducting wire by conductive silver paste plus (SPI, USA). The sample cell was composed of an ITO electrode and a glass slide $(25 \mathrm{~mm} \times 40 \mathrm{~mm}, 6 \mathrm{~mm}$ thick), and there was a hole with the diameter of $5 \mathrm{~mm}$ in the middle of the glass slide. The sample cell was constructed by gluing the ITO onto the glass slide via vacuum grease. 


\section{Fabrication Procedure of Biosensor}

A sandwich type ECL assay interface was fabricated on the aminated ITO slice by stepwise modification. As shown in Figure S2, $60 \mu \mathrm{L}$ of $5 \%$ GLD was placed onto the sample cell at room temperature for $60 \mathrm{~min}$. Then, $60 \mu \mathrm{L}$ of $0.1 \mu \mathrm{g} / \mathrm{mL} \mathrm{Ab}$, was introduced onto the GLD/ITO activated surface by covalent coupling. After washing with PBS, $60 \mu \mathrm{L}$ of $1 \%$ BSA was injected into the sample cell and left for $60 \mathrm{~min}$ at $37^{\circ} \mathrm{C}$. The resulting sample cell was then incubated with $60 \mu \mathrm{L}$ of CK19 solution at various concentrations at $37^{\circ} \mathrm{C}$ for $60 \mathrm{~min}$. Finally, $60 \mu \mathrm{L}$ of the RuDSN/AuNPs/Ab 2 was dropped onto the electrode and incubated at $37^{\circ} \mathrm{C}$ for $30 \mathrm{~min}$.

\section{Single-Molecule Fluorescence Imaging}

Consistent with the previous steps, the $\mathrm{Ab}_{1}$ modified ITO electrode was incubated with $60 \mu \mathrm{L}$ of $5 \mathrm{ng} / \mathrm{mL} \mathrm{CK} 19$ at $37^{\circ} \mathrm{C}$ for $1 \mathrm{~h}$. Successively, the sample cells were treated with $60 \mu \mathrm{L}$ of $0.5 \mu \mathrm{g} / \mathrm{mL}$ biotin-Ab $\mathrm{b}_{2}$ at $37^{\circ} \mathrm{C}$ for $2 \mathrm{~h}$. After being washed three times, the sample cells were added with $60 \mu \mathrm{L}$ of $200 \mathrm{pM}$ streptavidin-Cy5 solution and incubated at room temperature for $1 \mathrm{~h}$. Finally, the sample cells were added with $50 \mu \mathrm{L}$ of PBS buffer for single-molecule fluorescence imaging.

\section{Culture and Fix Cells on the ITO Electrode}

The MCF-7 (human breast adenocarcinoma) and L-02 (human normal hepatocytes) cell lines were obtained from Cell Bank of Chinese Academy of Sciences (Shanghai, China). The cells were cultured in DMEM medium supplemented with $10 \%$ FBS and $1 \%$ penicillin streptomycin at $37^{\circ} \mathrm{C}$ under a humidified atmosphere containing $5 \% \mathrm{CO}_{2}$. The cells in the exponential growth phase were digested for $2 \mathrm{~min}$ by trypsin $(0.1 \%, \mathrm{~m} / \mathrm{v})$, and then separated from the cell culture medium. Centrifuging at $800 \mathrm{rpm}$ for $3 \mathrm{~min}$ to gather the cells and resuspend in $10 \mathrm{~mL}$ of DMEM.

Prior to seeding cells, the ITO electrodes were etched in vacuum plasma (PDC-002, Harrick Plasma Inc., U.S.) for 5 min. Then, they were immersed into $0.01 \%(\mathrm{w} / \mathrm{v})$ PLL solution for a $10 \mathrm{~min}$ incubation and followed by washing thoroughly with water to remove excess solution, which can increase cell adhesion of the sensing interface. ${ }^{4}$ After that, living cells were seeded on the pole of the $\mathrm{PLL} / \mathrm{ITO}$ and incubated for $12 \mathrm{~h}$ with culture medium at $37^{\circ} \mathrm{C}, 5 \% \mathrm{CO}_{2}$. Before $\mathrm{ECL}$ measurement, the cells were fixed for $10 \mathrm{~min}$ with PFA (4\%), permeabilized for 10 min with Triton X-100 (0.1\%). After blocking the nonspecific binding sites on the cell and electrode surface with BSA (1\%) for $30 \mathrm{~min}$, the RuDSN/AuNPs/Ab 2 nanoprobes were introduced and incubated with fixed cells for $1 \mathrm{~h}$ at $37^{\circ} \mathrm{C}$ The ECL single cell imaging measurements were performed after washing the labeling cells with sterile PBS. For comparison, L-02 cells were seeded and labeled under the same conditions.

\section{ECL Detection and Imaging}

For conventional ECL intensity test, the working electrode (WE) was the modified ITO electrode, the auxiliary electrode (AE) was a $\mathrm{Pt}$ wire and the reference electrode (RE) was an $\mathrm{Ag} / \mathrm{AgCl}$. The $\mathrm{ECL}$ experiments were carried out in PBS $(0.1 \mathrm{M}, \mathrm{pH} 7.4)$ containing $0.1 \mathrm{M}$ TPA, and the light/current/voltage curves were recorded by collecting the preamplified PMT output signal with an MPI-M ECL analyzer (Xi'An Remax Electronic Science \& Technology Co., Ltd., Xi'An, China).

For ECL imaging, a voltage generator (DG 1021, Rigol, China) connected in a two-electrode system was used to facilitate the application of the voltage with a different frequency at ITO slide (Figure S3). In our work, a switching of the potential between $+1.35 \mathrm{~V}$ $(2 \mathrm{~s})$ and $-1 \mathrm{~V}(0.5 \mathrm{~s})$ was continuously employed on the ITO electrode to provide the needed potential or the ECL triggered reaction. Then, a wide-field epi-fluorescence microscope from Nikon (Chiyoda, Tokyo, Japan) equipped with ultrasensitive Electron-Multiplying CCD camera (EM-CCD, Ixon DU897, Andor Technology Plc., U.K.) was used to collect the optical signal. Besides, the singlemolecule detection platform was equipped with a motorized microscope stage for sample positioning and an oil immersion objective (NA 1.45, 100x, Olympus, Japan). For the acquisition of single-molecule data, 10 regions were imaged of each sample. The MATLAB (MathWorks, MA, U.S) program was used to analyse the single-molecule data. For each frame, the interest region with a size of $40 \times$ $20 \mu \mathrm{m}$ was employed to count the image spots.

\section{Data Analysis Process for Single Molecule Fluorescence Imaging}

The data analysis method is implemented from the Professor W.E. Moerner lab to recognize single molecule spots in images with some modification. This method that is written in the MATLAB program first converts the raw image tiff file into black-white bit image with a threshold number. This threshold determines one pixel to become 1 (namely white pixel) if its intensity is above the threshold or to be 0 (namely black pixel) if not. Then a MATLAB function is used automatically to identify all white-segments that are composed of white pixels and also all the white pixels of one segment are connected each. In other words, the MATLAB function guarantees that one segment would not include multiple separated white fragments. Each white pixel segment corresponds to one bright spot The MATLAB function also obtains the column/row-wise coordinates of pixels in every segment. By using such coordinate information, the program could determine the center coordinate of every spot and then choose a range of pixels in the raw tiff image 
around the center to do 2D gaussian fit. Such gaussian fit would calculate the key parameters of a spot: accurate center coordinates and FWHMs along both row and column directions.

A strategy named waterfall filtering was implemented in the MATLAB program to count the image spots. The program first starts with a very high threshold that means only a few very bright spots can be recognized. The program determines all the parameters of these bright spots as we describe in above. Then the program deletes these bright spots in the raw image by replacing the pixels of these spots with background level brightness intensities. Subsequently, the program uses a lower threshold to process the modified image again and some less bright spots are recognized this time. Iteratively, the program keeps deleting the already recognized spots and lowing the threshold to recognize less bright spots until the threshold reaches the level of background.

Also, the program would determine if the size of a spot is below the diffraction limit by comparing the fitting FMHM and a criteria Those unqualified spots with too large diameters are discard. The qualified spots then would be marked on the tiff image. The program usually can recognize all the spots very well, but the researcher would check every set of images and discard some lowquality spots. The researcher can also pick up those spots missed by the program through approximately locate their centers that would be calculatedly accurately by the program. Usually we manually change few spots.

If we need to get the brightness intensity trace of spots, the program can add up the intensity of one spot's pixels within an octagon around its center with an approximate diameter of the FMHM and substrate the corresponding background intensity. With the combination of automatic processing of images, the waterfall method that identifies spots in the order of successive batches and the manual check of spots, our method recognizes spots accurately even under the condition of high spot density.

\section{Estimation of the Average Number of Dyes per NP}

The average number of dyes embedded in the NP was calculated according to the previously reported ${ }^{5-7}$

First we calculate the mean volume $\left(V_{N P}\right)$ of a single (spherical) RuDSN from the diameter $(60 \mathrm{~nm})$ measured by TEM images in Figure S1

$$
V_{N P}=\frac{4}{3} \pi\left(30 \times 10^{-8}\right)^{3}=1.1 \times 10^{-19} \mathrm{dm}^{3}
$$

By the tabulated $\mathrm{SiO}_{2}$ density $\left(\delta_{N P}\right)\left(2.2 \mathrm{Kg} / \mathrm{dm}^{3}\right)$, we obtain that the mean weight of a single RuDSN $\left(W_{N P}\right)$ is

$$
W_{N P}=V_{N P} \times \delta_{N P}=2.4 \times 10^{-19} \mathrm{Kg}
$$

By dividing $W_{N P}$ for the $\mathrm{SiO}_{2}$ molecular weight (60 UMA), we find that the number of silica molecules per NP ( $n_{\mathrm{SiO} / \mathrm{NP}}$ ) are

$$
n_{\mathrm{SiO} 2 / N P}=\frac{W_{N P}}{60 \times 1.67 \times 10^{-27}}=2.4 \times 10^{6}
$$

The dye molecules per NP ( $\left.n_{\text {dye/NP }}\right)$ that can be estimated from the value of RuDSN doping ratio, that in this case was of $1.5 \%$ moles

$$
n_{d y e / N P}=1.5 \% \times n_{S i O 2 / N P}=3.6 \times 10^{4}
$$




\section{Supplementary Figures}

S1. Characterization of the Nanoemitter


Figure S1. (A) SEM image of RuDSNs. (B) TEM image of RuDSNs. (C) Size distribution of RuDSNs observed from TEM images. (D) TEM image of RuDSN/AuNPs.

S2. ECL Enhancement from RuDSN in presence of AuNPs

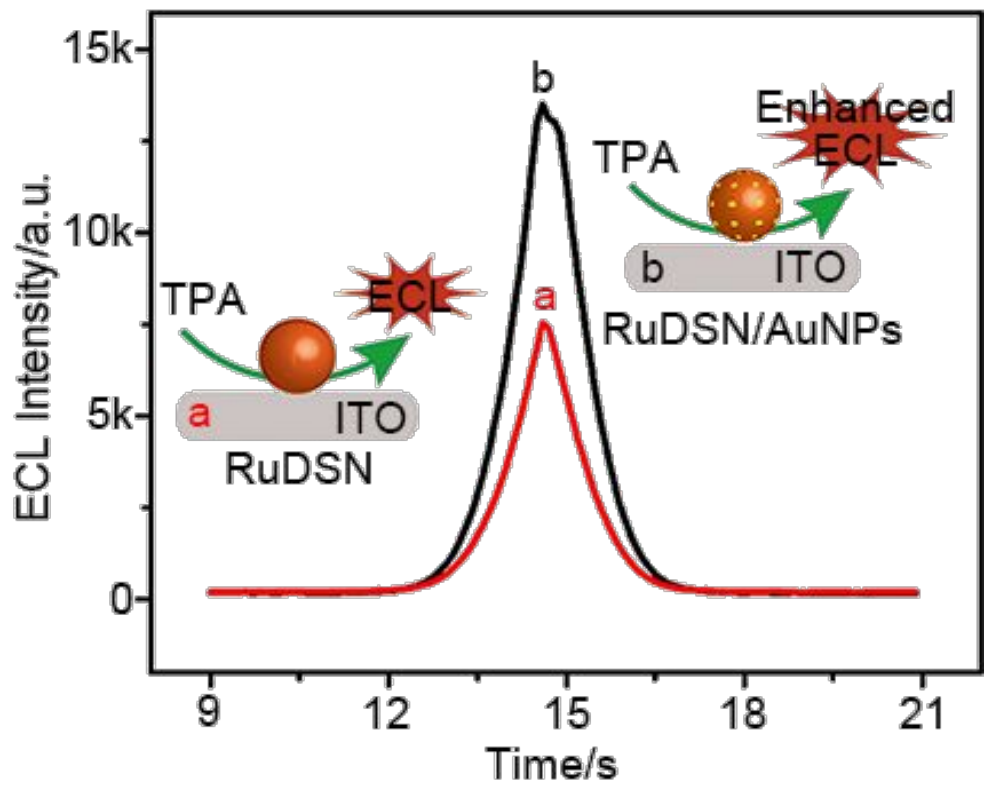

Figure S2. The ECL enhancement from RuDSN in presence of AuNPs. Electrolyte: 0.1 M PBS (pH 7.4) containing $10 \mathrm{mM}$ TPA; scan rate: $100 \mathrm{mV} / \mathrm{s}$; scan potential: $0-1.35 \mathrm{~V}$. The voltage of the PMT was set at $800 \mathrm{~V}$. 
S3. Scheme Illustration for the Single Protein ECL Imaging



Figure S3. Schematic illustration for the single protein ECL imaging.

S4. Electrochemical and ECL Response of the Nanoemitter
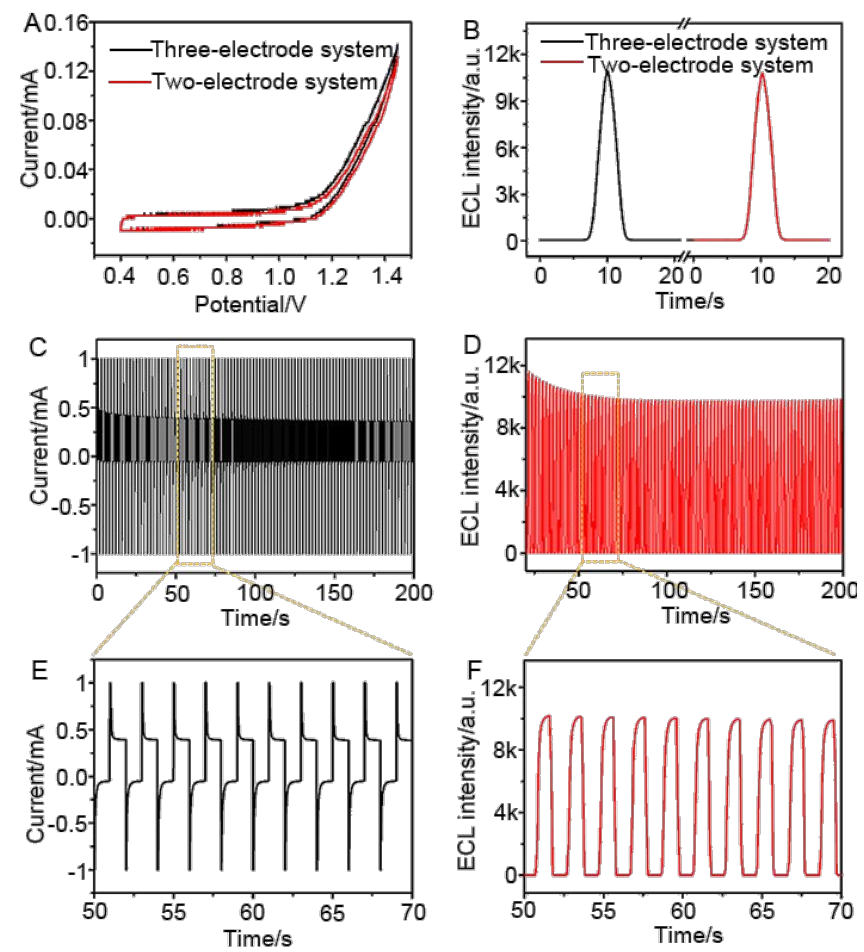

Figure S4. (A) The current and (B) ECL intensity collected from ITO electrode using three-electrode and two-electrode system. (C,E) Chronoamperometry response and (D,F) ECL intensity using two-electrode system. Chronoamperometry technique was used for $E C L$ detection: Initial voltage $=0 \mathrm{~V}$, high voltage $=1.4$ $\mathrm{V}$, low voltage $=0 \mathrm{~V}$, pulse time $=1 \mathrm{~s}$. The voltage of the PMT was set at $700 \mathrm{~V}$. 


\section{S5. Voltage Optimization}
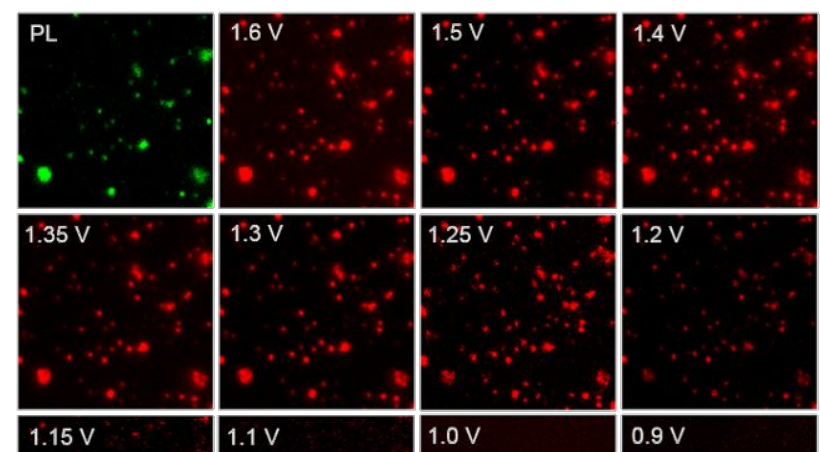

$1.0 \mathrm{~V}$

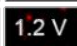

$1.15 \mathrm{~V}$

$1.1 \mathrm{~V}$

$0.9 \mathrm{~V}$
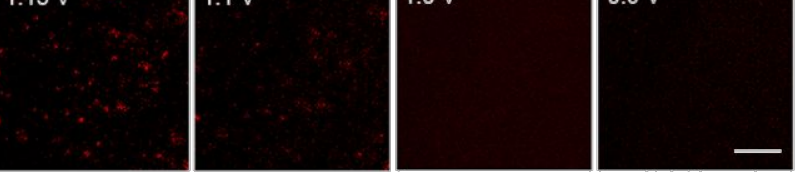

Figure S5. ECL images of the nanoemitter with different potentials in $0.1 \mathrm{M}$ PBS containing $0.1 \mathrm{M}$ TPA Scale bar: $5 \mu \mathrm{m}$.

\section{S6. Frequency Optimization}
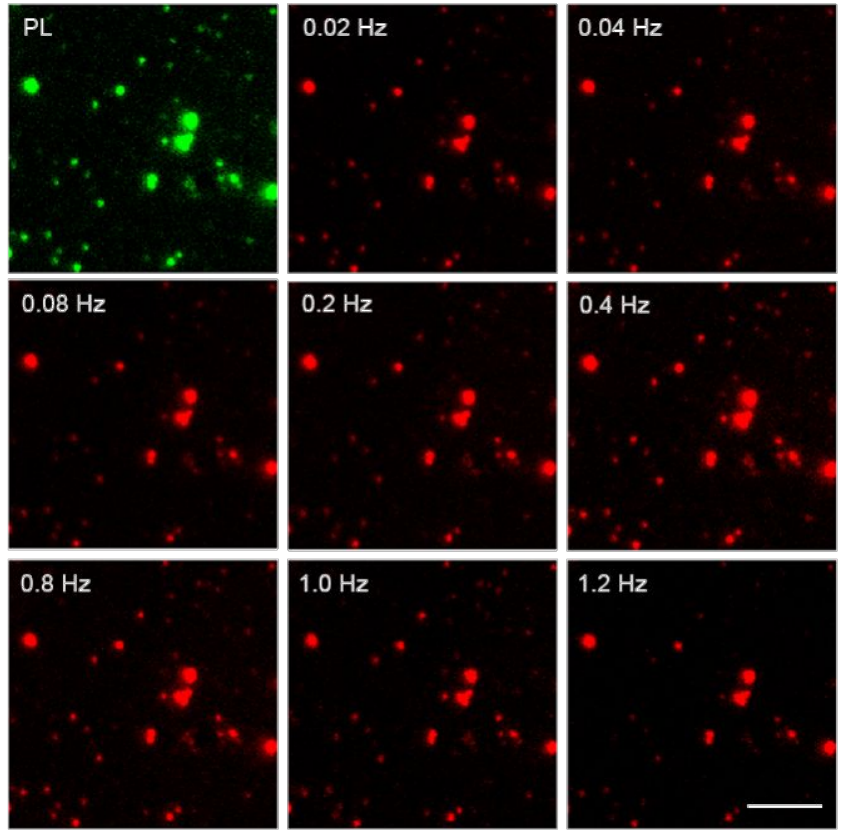

Figure S6. ECL images of the nanoemitter with different potential output frequencies in $0.1 \mathrm{M}$ PBS containing $0.1 \mathrm{M}$ TPA. Scale bar: $5 \mu \mathrm{m}$. 


\section{S7. Exposure Time Optimization}

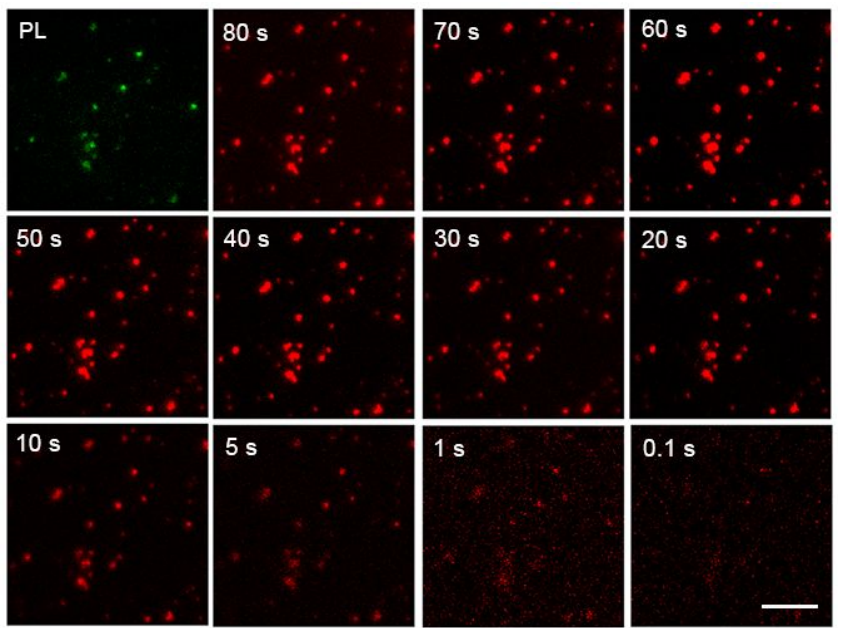

Figure S7. ECL images of the nanoemitter with different exposure times in $0.1 \mathrm{M}$ PBS (pH 7.4) containing $0.1 \mathrm{M}$ TPA. Scale bar: $5 \mu \mathrm{m}$.

S8. Size Analysis of PL and ECL spots
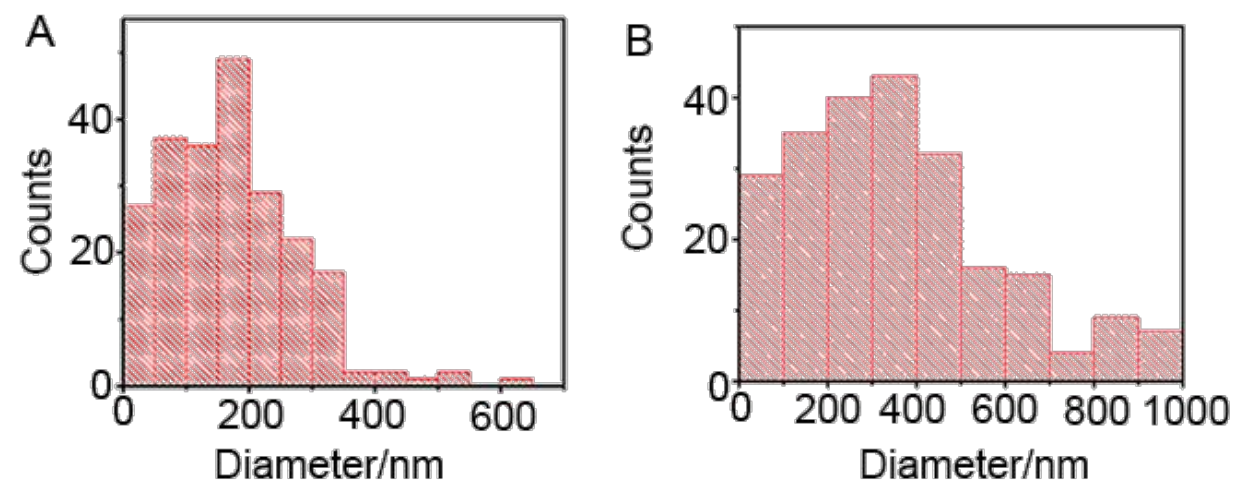

Figure S8. Diameter distribution of $(A)$ the $P L$ spots from individual Cy5-Ag; $(B)$ the $E C L$ spots from individual nanoemitter-Ag. 
S9. The AFM image of ECL nanoemitters
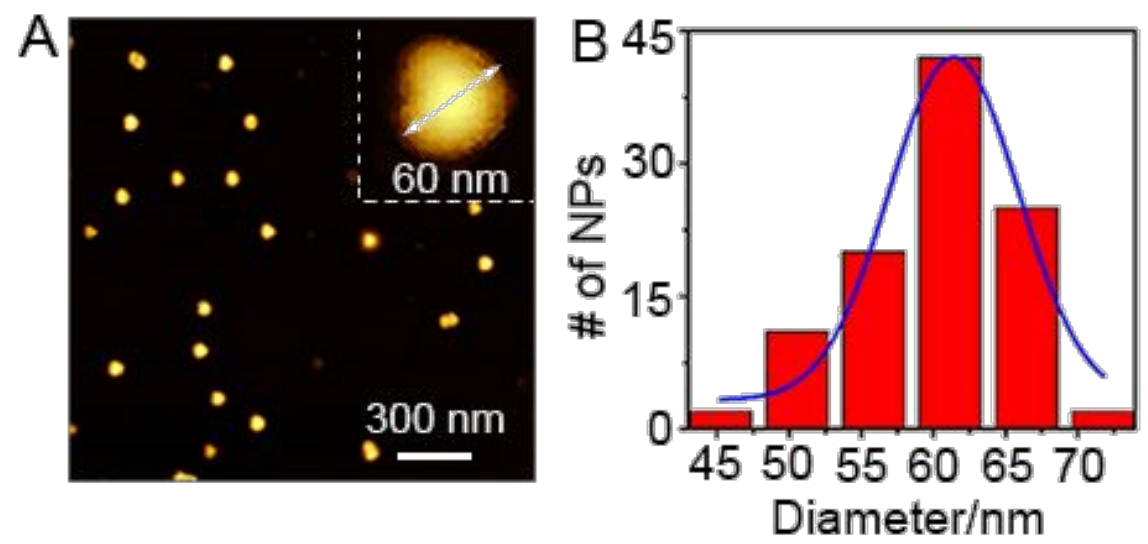

Figure S9. (A) The AFM images of RuDSNs. (B) Size distribution of RuDSNs observed from AFM images.

S10. The $S / N$ ratios of $E C L$ and $P L$ from single antigen
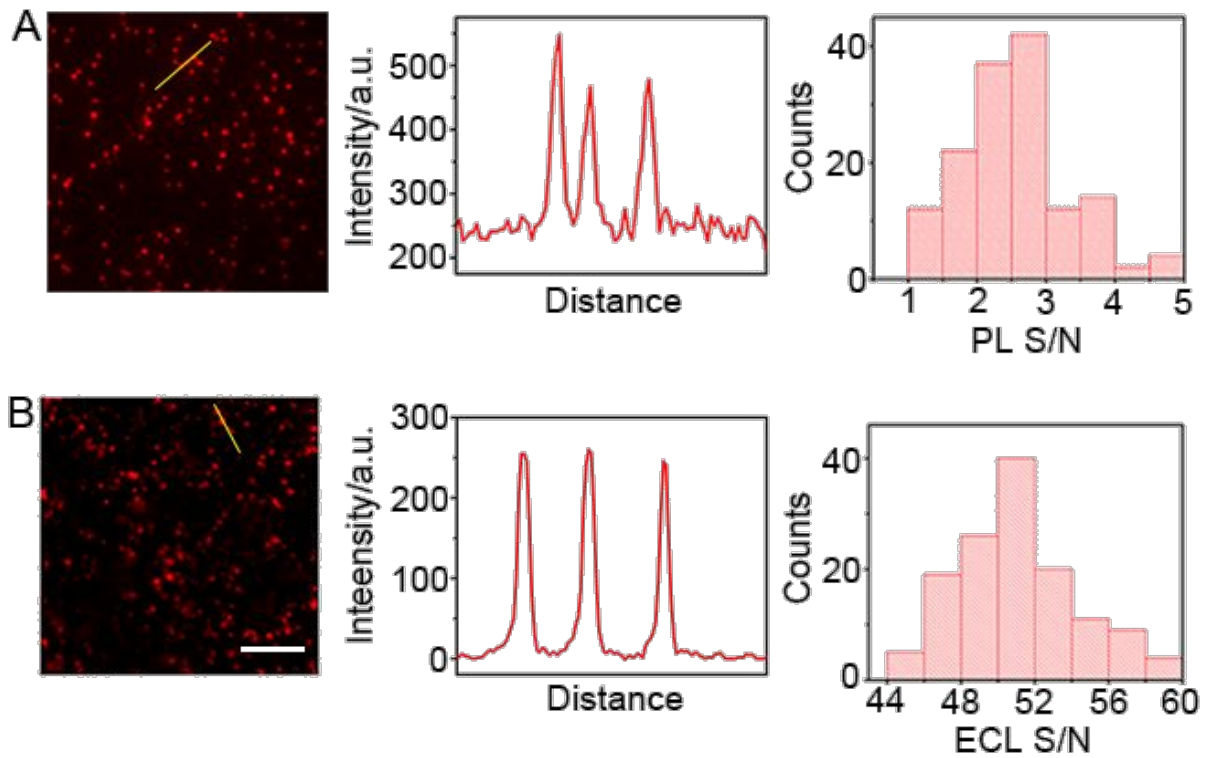

Figure S10. (A) PL image, PL profiles along the yellow line and distribution of $S / N$ of PL emission for about 150 Cy5 labeled antigens. (B) ECL image, ECL profiles along the yellow line and distribution of S/N of ECL emission for about 150 nanoemitters labeled antigens. Scale bar: $5 \mu \mathrm{m}$. 


\section{S11. Stablity of ECL from single antigen}

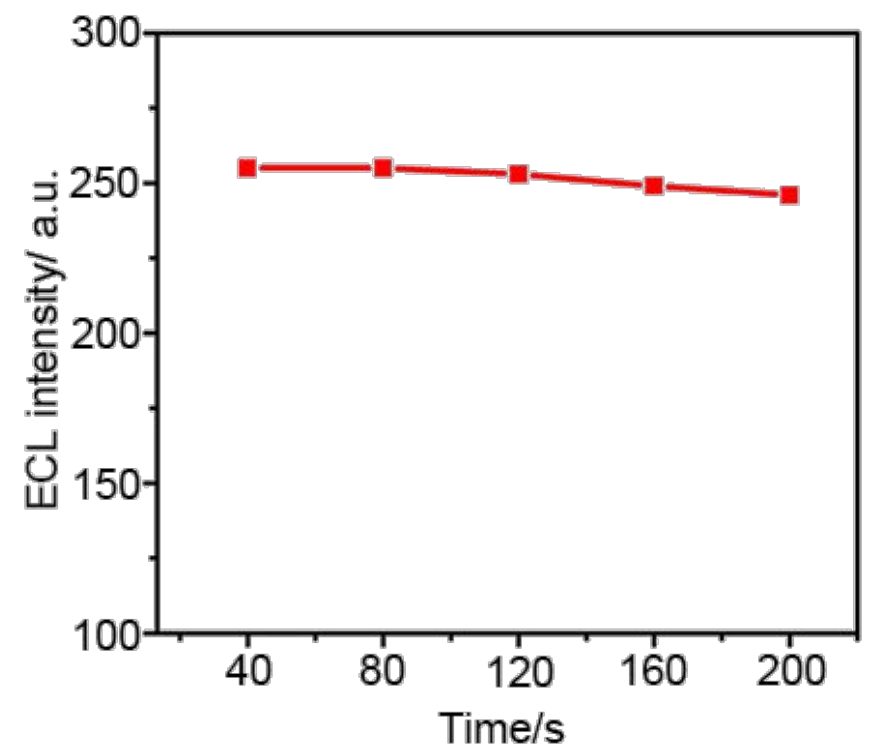

Figure S11. The ECL intensity collected from one RuDSN/AuNPs/Ab ${ }_{2}-\mathrm{Ag}$ complex in $200 \mathrm{~s}$. The exposure time is $40 \mathrm{~s}$

\section{S12. Reproducibility and Stability of the Proposed Method}

To investigate the reproducibility, five sample cells for $0.2 \mathrm{ng} / \mathrm{mL}$ CK19 of same conditions (inter-assay), and repeat 5 times in one sample cell (intra-assay) were evaluated. The relative standard deviations (RSDs) of inter-assay and intra-assay are $5.5 \%$ and $3.4 \%$, indicating acceptable precision and fabrication reproducibility. The stability was tested by monitoring the ECL counts of CK19 at different concentrations (0.02 ng/mL, $0.04 \mathrm{ng} / \mathrm{mL}, 0.2 \mathrm{ng} / \mathrm{mL}, 0.4 \mathrm{ng} / \mathrm{mL}$, and $1 \mathrm{ng} / \mathrm{mL}$ ). As shown in Figure S10B, the method has good stability by multiple measurements over a long period of time with RSDs of $6.0 \%, 4.8 \%, 4.1 \%, 4.0 \%$, and $3.6 \%$, respectively.
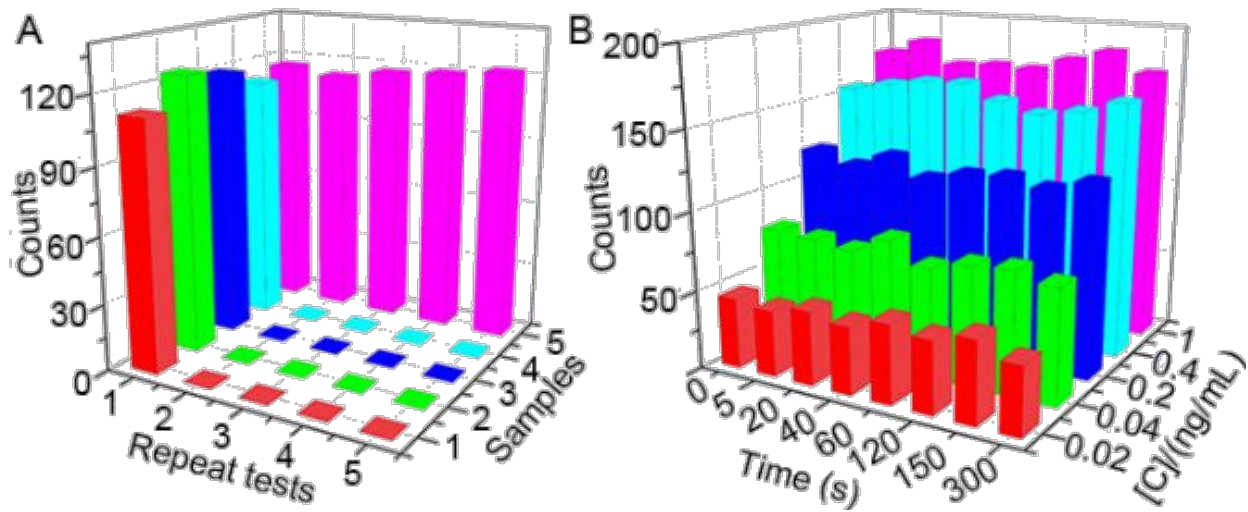

Figure S12. (A) Reproducibility and (B) stability of the proposed method. 


\section{Table S1. Detection of CK19 in Human Serum}

We apply the proposed ECL microscopy to measure the CK19 concentration in human serum samples by spiking varied concentrations of CK19. The results listed in Table S1 demonstrate that the recoveries are in the range of $96.8 \%$ to $103.9 \%$, and the RSD of three parallel experiments is no more than $5.7 \%$, which indicated that the design shows good accuracy for CK19 detection in real biomedical samples.

\begin{tabular}{cccc}
\multicolumn{4}{c}{ Table S1. Detection of CK19 in Human Serum. } \\
\hline $\begin{array}{c}\text { Add } \\
(\mathrm{pg} / \mathrm{mL})\end{array}$ & $\begin{array}{c}\text { Found } \\
(\mathrm{pg} / \mathrm{mL})\end{array}$ & $\begin{array}{c}\text { Recovery } \\
(\%)\end{array}$ & $\begin{array}{c}\text { RSD }(\%) \\
(\mathrm{n}=3)\end{array}$ \\
\hline 20 & 19.7 & 98.5 & 2.7 \\
100 & 101.5 & 101.5 & 5.2 \\
1000 & 968.0 & 96.8 & 4.1 \\
5000 & 5194.3 & 103.9 & 5.7 \\
\hline
\end{tabular}

Figure S13. The $\mathrm{S} / \mathrm{N}$ ratios of ECL and $\mathrm{PL}$ from the antigens at single cells
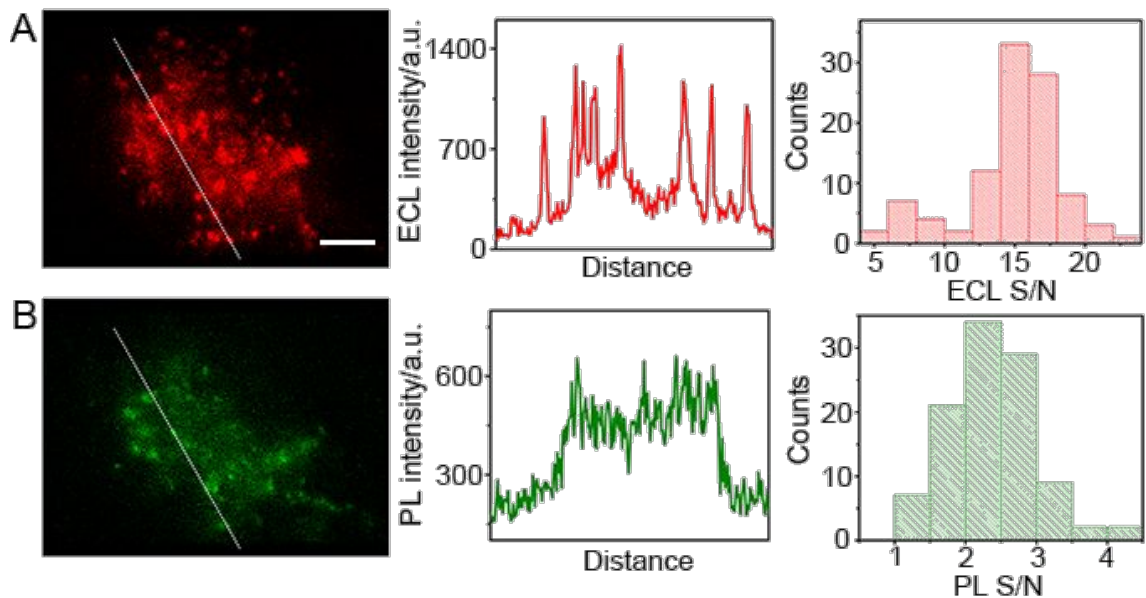

Figure S13. (A) ECL image, ECL profiles along the white line and distribution of $S / N$ of ECL emission for about 150 nanoemitters on cell membrane. (B) PL image, PL profiles along the white line and distribution of $\mathrm{S} / \mathrm{N}$ of ECL emission for about 150 nanoemitters on cell membrane. Scale bar: $5 \mu \mathrm{m}$. 


\section{References}

(1) Zhang, L.; Dong, S. Electrogenerated chemiluminescence sensors using doped in silica nanoparticles. Anal. Chem. 2006, 78, 5119-5123.

(2) Cao, J.-T.; Wang, Y.-L.; Zhang, J.-J.; Dong, Y.-X.; Liu, F.-R.; Ren, S.-W.; Liu, Y.-M. Immunoelectrochemiluminescent imaging of a single cell based on functional nanoprobes of heterogeneous $\mathrm{Ru}(\mathrm{bpy})_{3}{ }^{2+} @ \mathrm{SiO}_{2} / \mathrm{Au}$ nanoparticles. Anal. Chem. 2018, 90, 10334-10339.

(3) Zhang, W.; Xiong, H.; Chen, M.; Zhang, X.; Wang, S. Surface-enhanced molecularly imprinted electrochemiluminescence sensor based on $\mathrm{Ru} @ \mathrm{SiO}_{2}$ for ultrasensitive detection of fumonisin $\mathrm{B}_{1}$. Biosens. Bioelectron. 2017, 96, 55-61.

(4) Xu, H.; Liao, C.; Liu, Y.; Ye, B.-C.; Liu, B. Iron phthalocyanine decorated nitrogen-doped graphene biosensing platform for real-time detection of nitric oxide released from living cells. Anal. Chem. 2018, 90, 4438-4444.

(5) Zanarini, S.; Rampazzo, E.; Ciana, L. D.; Marcaccio, M.; Marzocchi, E.; Montalti, M.; Paolucci, F.; Prodi, L. $\mathrm{Ru}(\mathrm{bpy})_{3}$ covalently doped silica nanoparticles as multicenter tunable structures for electrochemiluminescence amplification. J. Am. Chem. Soc. 2009, 131, 2260-2267.

(6) Valenti, G.; Rampazzo, E.; Bonacchi, E.; Petrizza, L.; Marcaccio, M.; Prodi, L; Paolucci, F. Variable doping induces mechanism swapping in electrogenerated chemiluminescence of $\mathrm{Ru}(\mathrm{bpy})_{3}{ }^{2+}$ core-shell silica nanoparticles. $J$. Am. Chem. Soc. 2016, 138, 15935-15942.

(7) Zanut, A.; Palomba, F.; Scota, M. R.; Rebeccani, S.; Marcaccio, M.; Genovese, D.; Rampazzo, E.; Valenti, G.; Paolucci, F.; Prodi, L. Dye-doped silica nanoparticles for enhanced ECL-based immunoassay analytical performance. Angew. Chem. Int. Ed. 2020, 59, 21858-21863.

(8) Preus, S.; Noer, S.L.; Hildebrandt, L.L.; Gudnason, D.; Birkedal, V. iSMS: single-molecule FRET microscopy software. Nat. Methods 2015, 12, 593-594. 